\title{
Fragilité urbaine des petites villes-mines : le cas d'Épinac, Saône-et-Loire
}

Urban fragility of the small mining towns : Épinac example, Saône-et-Loire

Die städtische Brüchigkeit der kleinen Bergwerk-städte: das Fallbeispiel von

Epinac, Saône-et-Loire

\section{Loman Gauduchon}

\section{(2) OpenEdition}

Journals

Édition électronique

URL : http://journals.openedition.org/rge/9243

DOI : $10.4000 /$ rge. 9243

ISSN : 2108-6478

Éditeur

Association des géographes de l'Est

Référence électronique

Loman Gauduchon, «Fragilité urbaine des petites villes-mines : le cas d'Épinac, Saône-et-Loire », Revue Géographique de l'Est [En ligne], vol. 58/3-4 | 2018, mis en ligne le 06 juillet 2020, consulté le 08 septembre 2020. URL : http://journals.openedition.org/rge/9243 ; DOI : https://doi.org/10.4000/rge. 9243

Ce document a été généré automatiquement le 8 septembre 2020

Tous droits réservés 


\title{
Fragilité urbaine des petites villes- mines : le cas d'Épinac, Saône-et- Loire
}

\author{
Urban fragility of the small mining towns : Épinac example, Saône-et-Loire \\ Die städtische Brüchigkeit der kleinen Bergwerk-städte: das Fallbeispiel von \\ Epinac, Saône-et-Loire
}

Loman Gauduchon

1 Si la littérature géographique en matière de développement, reconversion et héritage industriels est aujourd'hui bien pourvue, celle-ci s'est principalement concentrée sur l'analyse des grands centres urbains et leurs faubourgs industriels (Laferrère, 1960 ; Bruyelle et Dézert, 1983 ; Edelblutte, 2009) où l'observation minutieuse de villes-usines emblématiques, en premier lieu celles aux origines planifiées et abouties, constituent les modèles les plus éminents (Edelblutte et Del Biondo, 2016), de toute nature et de toute échelle (Doyen, 1983 ; Edelblutte, 1998, 2014 ; Dorel-Ferré, 2003, 2016).

2 Par leurs inspirations souvent influencées d'utopies urbaines, d'hygiénisme ou de considérations plus politiques, ces modèles de développement et leur morphologie induite, produits par l'essor des diverses activités industrielles, ont constitué un objet d'étude particulièrement riche et aujourd'hui densément documenté. Ceci qu'il s'agisse d'études empiriques ou d'approches systémiques, balayant les différentes échelles et aires géographiques de ces systèmes productifs (Edelblutte, 2010).

Devant un tel foisonnement l'étude des systèmes extractifs et productifs de dimension plus modeste (souvent à la lisière entre proto-industrie de l'époque moderne et premières villes-usines et géosystèmes ${ }^{1}$ industriels nés de la première révolution industrielle), demeure bien moins fournie (Doumergue, 1964). Souvent inscrits au sein de milieux ruraux, ces lieux ont fait l'objet d'analyses concentrées sur les héritages induits par l'industrialisation de ces territoires (Edelblutte et Legrand, 2012, DorelFerré, Varashin et al., 2016), davantage qu'à leur genèse et leur essor proprement dits. 
4 L'étude du développement proto-industriel, puis industriel, en tant qu'objet géographique et appréhendé dans l'ensemble de son processus de développement systémique et productiviste, dispose ainsi d'une littérature et d'une visibilité scientifique moindre, eu égard à la diversité des cas et lieux disponibles en la matière pour étayer le sujet (Woronoff, 1998).

5 L'étude qui suit a donc pour objectif de montrer, par l'exemple de la genèse à la crise puis la disparition des activités minières et industrielles apparues à Épinac (BourgogneFranche-Comté), comment et pourquoi un géosystème industriel planifié en milieu rural, pensé et conçu par ses administrateurs comme une véritable ville en devenir, n'aboutit pas de manière pérenne au modèle de ville-usine.

6 Il s'agit ainsi de questionner le dimensionnement et la taille urbaine critique d'une ville-usine, ici par l'exemple d'une ville-mine (Epinac) et ainsi d'explorer, par-delà les modèles urbains nés de l'industrie, les contours plus flous et mal-définis de territoires industriels et miniers, dont le développement incomplet ou modeste aboutit à des formes moins bien connues de petites villes-usines et petites villes-mines. Ces territoires n'en demeurent pas moins confrontés aujourd'hui à une crise urbaine similaire en certains points aux problématiques qui caractérisent les «shrinking cities $»^{2}$.

7 La ville-usine se définit comme une ville créée en tout ou quasi-totalité par et pour l'usine (Edelblutte, 2010). À la différence de la ville industrielle, dont le fait urbain préexistait à l'essor industriel et dont le caractère urbain n'a été qu'amplifié par l'émergence d'un quartier industriel, le plus souvent à sa périphérie, la ville-usine possède la particularité d'entretenir un lien consubstantiel entre son urbanité et son tissu industriel.

8 Le modèle s'adapte également aux contextes d'exploitation minière, avec le terme équivalent de ville-mine (ou coke-town en anglais), et désigne ainsi des agglomérations ayant atteint, du fait de leur activité industrielle ou minière, une taille urbaine critique. Outre l'organisation de la production et de ses annexes et les infrastructures qui y sont directement liées, la ville-mine fonctionnelle se caractérise également par l'impact prépondérant de l'activité minière sur un grand nombre de réalisations indirectes : logements, édifices culturels, cultuels, sociaux... (Del Biondo, Edelblutte, 2016).

${ }^{3}$ ex-nihilo I

9 Ainsi, d'autres facteurs sont intervenus dans l'affirmation ou, a contrario, la dégénérescence d'un géosystème industriel en milieu rural et de l'identité urbaine que celui-ci tend à affirmer. Ces facteurs, multiples, ont constitué de puissants freins à l'essor d'Épinac en tant que véritable ville-mine d'une part, mais ont également contribué à la disparition progressive de l'activité industrialo-minière du bourg d'autre part, et ainsi à divers questionnements quant aux reconversions des sites et du système productif dans son ensemble (II). 
Figure 1 : Épinac au sein de son espace régional

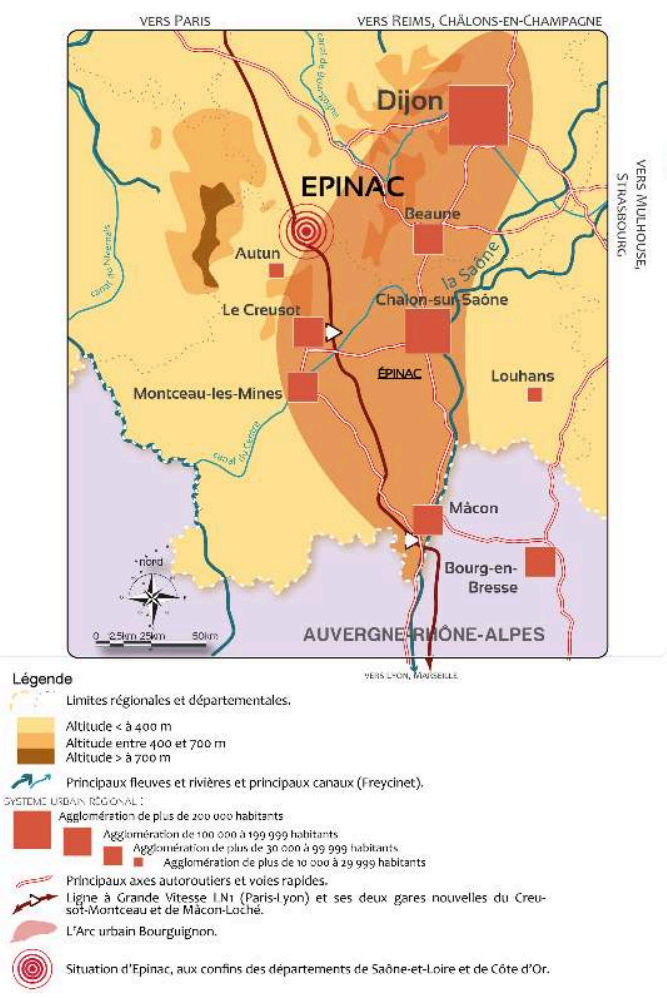

\section{I. Épinac : ville duale ou ville-mine?}

Après avoir mis en évidence l'ensemble des éléments industriels et paternalistes qui ont amené à bâtir une ville-mines (A), une mise en perspective complémentaire permettra de souligner les limites de ce développement dans son l'affirmation de son caractère urbain, notamment par la préexistence d'un bourg rural d'époque médiévale (B).

\section{A. Épinac, de la proto-industrie au géosystème industriel paternaliste}

D'un point de vue géographique et géologique, Épinac se situe sur la bordure nord du Massif central, sur les premiers contreforts orientaux de l'appendice orographique que constitue le Morvan (Figure 1). En périphérie orientale du bassin géologique d'Autun et rattaché aux formations hercyniennes du Massif central, le site d'Épinac compte parmi les gisements houillers les plus septentrionaux de cette formation géologique du centre de la France.

Elle s'inscrit ainsi dans le prolongement du bassin de Blanzy, dont les grands centres industrialo-urbains du Creusot et de Montceau-les-Mines constituent encore aujourd'hui l'un des principaux bassins industriels de la région (métallurgie, sidérurgie, verrerie, exploitation minière).

Progressivement développé autour du vieux bourg médiéval d'Épinac à partir du XVIII siècle, le tissu industrialo-minier d'Épinac s'est constitué en plusieurs étapes. D'abord 
tourné autour de la seule usine verrière, négligeant l'ensemble des autres sphères productives, l'activité industrielle connaît durant le XIX ${ }^{\mathrm{e}}$ siècle un essor décisif.

Par la conglomération des activités (verrerie, houillères, chemin de fer) ainsi que par un déploiement d'actions de type paternaliste dans l'ensemble des domaines touchant à l'urbanisme, la vie sociale et économique de la cité minière (nouveaux quartiers de logements, écoles, église, marché, coopératives, etc), l'exploitation houillère à Épinac aboutit au développement d'un nouveau tissu urbain entièrement né de ce développement des systèmes productifs locaux et indépendant du précédent bourg d'Ancien Régime.

Ce nouveau tissu urbain comprend, d'une part ce qui est généré directement par l'usine ou le site d'exploitation minière et ses sites de productions (chevalements, puits, lavoirs, triages, etc) et, d'autre part, les productions urbaines annexes que peut induire l'implantation industrielle (logements, œuvres sociales, alimentaires, etc). Le tout, plus ou moins dense, mais intimement lié à l'activité industrielle et minière, constitue un ensemble cohérent et aux logiques d'organisation quasi endogène, ainsi assimilable sous le terme de géosystème industriel.

Un premier géosystème, en prolongement direct du village, se développe sur le territoire communal dès la seconde moitié du XVIII ${ }^{e}$ siècle sous l'impulsion des propriétaires de l'industrie verrière. Incomplet, minimaliste et sans recherche d'action sociale d'ampleur, ce géosystème est à l'image de son site industriel central, la verrerie. Né de l'ère proto-industrielle, et encore modérément marquée par les idées et considérations hygiénistes caractéristiques aux industriels paternalistes du milieu du XIXe siècle, il constitue néanmoins le premier véritable site industriel d'Épinac.

Ce géosystème, situé immédiatement à la périphérie de l'ancien bourg médiéval (Figure 2) demeure sommaire, modestement constitué du site de production de la verrerie, auquel se greffent au début du XIX siècle les casernes ouvrières de la rue dite "Bouteille ». Six bâtiments répartis géométriquement le long de la rue constituent ainsi l'ensemble d'habitations destiné aux ouvriers de la verrerie.

Si la direction de la verrerie a développé un géosystème minimaliste, ne répondant in fine qu'à la nécessité croissante de logements engendrée par son activité et à laquelle ne pouvait répondre le modeste parc de logements du bourg ancien, les houillères ont elles marqué d'une toute autre empreinte le tissu urbain et les paysages de la commune.

Effectivement, à la marge du tissu urbain alors existant au début du XIXe siècle, la compagnie minière d'Épinac développe progressivement un ensemble d'infrastructures liées à l'exploitation houillère : bâtiments industriels et miniers, voies de chemin de fer, gare et halte, habitats ouvriers, bâtiments culturels et d'activités sociales, etc. (Simonin, 1867). 
Figure 2 : Épinac, de l'essor industrialo-minier aux nouvelles logiques d'organisation du territoire

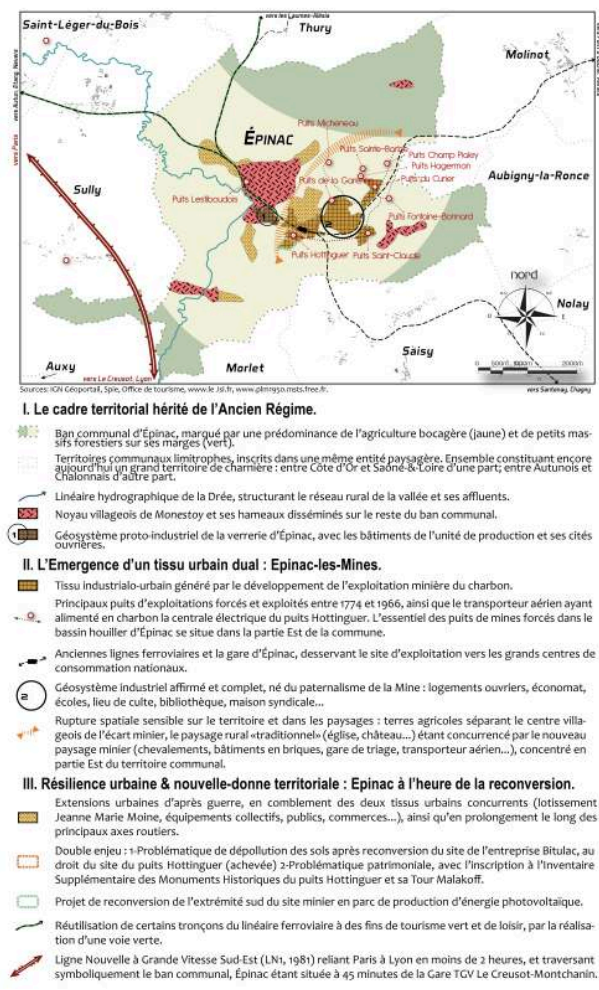

20 La compagnie lance ainsi dès 1834, à proximité du principal carreau minier de la concession, la réalisation d'une cité ouvrière au lieu-dit de la Garenne, en disjointe du tissu urbain villageois. Située à plus d'un kilomètre à l'est du noyau ancien, la Cité de la Garenne abrite, dès 1836, 92 logements ouvriers, puis 126, composant la "Vieille cité » (Figure 3). Comme l'illustre le schéma de la Figure 2, à ces 92 logements correspondants à la «Vieille cité » de 1836 se greffent 220 logements construits en 1864. L'ensemble de la Cité de la Garenne se compose de 6 types de maisons, et autant de variantes tant architecturales que d'aménagements intérieurs, sur près de 16 hectares (Blanchet, 1876).

21 In fine, ce sont ainsi 525 logements ouvriers qui composent la Cité de la Garenne au début du $\mathrm{XX}^{\mathrm{e}}$ siècle. L'organisation spatiale respecte, comme dans un grand nombre de cités ouvrières en Europe, la hiérarchie sociale de l'entreprise. Ainsi, les maisons de la Direction sont placées à l'écart de la cité ouvrière. Celles des employés et des maîtresmineurs sont construites à partir de 1846 et sont positionnées à la marge de la cité, mais en continuité immédiate des quartiers ouvriers, dans un souci d'encadrement des ouvriers et mineurs. La partie centrale de la Cité accueille quant à elle l'ensemble des logements ouvriers et les services qui leurs sont directement dédiés.

22 A l'instar de réalisations paternalistes étudiées dans des villes-usines de taille plus importante (Edelblutte, 2014), le bâti social est particulièrement développé au sein de ce géosystème. Aux constructions des logements ouvriers de la Cité de la Garenne se mêle une multitude d'édifices à caractère public: une infirmerie, une cantine, une caserne pour célibataires, des boulangeries, des écoles, une chapelle, plusieurs économats, une maison syndicale... 
Figure 3 : Morphologie urbaine de la cité minière de la Garenne à Epinac
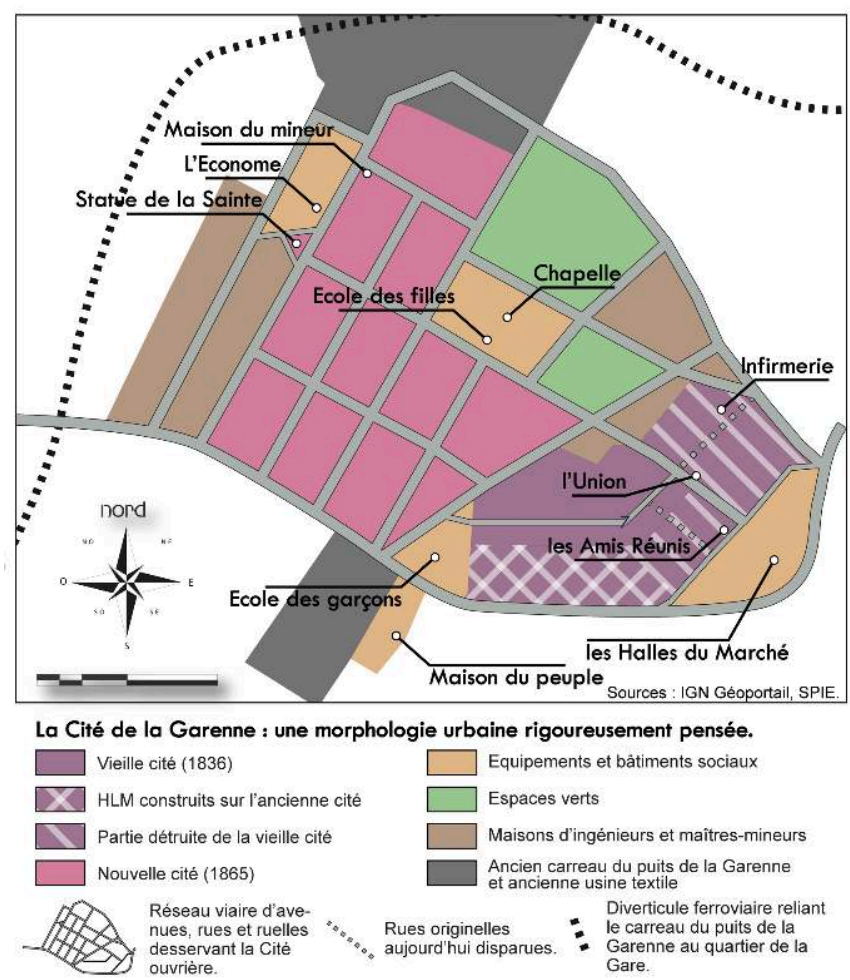

La cité ainsi constituée atteint près de 2500 habitants au plus fort de l'activité (fin XIX - début $\mathrm{XX}^{\mathrm{e}}$ siècle) et fait de ce géosystème à lui seul une véritable petite ville-mine fortement planifiée. Venant ainsi à dépasser la population du bourg originel, la population totale de la commune oscille entre 4000 et 4500 habitants à la charnière des XIX ${ }^{e}$ et XX $X^{e}$ siècles.

Qu'il s'agisse de l'importance du nombre de logement et des réalisations paternalistes des houillères, l'étendue des domaines que celles-ci recouvrent ou encore le bond démographique que celles-ci ont permis d'opérer pour la commune d'Epinac, ceci aboutit à confondre le géosystème minier d'Épinac dans les traits de modèles urbains connus, davantage en tout cas qu'à celui de village-usine (ou village-mine), ce dernier se démarquant par un développement fortement incomplet, lacunaire, peu ou prou doté d'équipements annexes ou indirects au site de production lui-même.

Cependant, par sa morphologie, le tissu urbain d'Épinac s'inscrit avant tout dans la logique d'une ville duale (Figure 2), entre vieux noyau à l'ouest et complexe industrialominier, auquel s'adosse la nouvelle Cité de la Garenne, plus à l'Est. Cette dualité constituant un point essentiel des enjeux et tensions de développement urbain de la commune depuis le XIX ${ }^{\mathrm{e}}$ siècle.

\section{B. Ville-mine et paysages industriels : Épinac, un géosystème minier ambivalent}

L'essor de l'activité minière et de ses annexes, excentrées par rapport au bourg médiéval, a construit le tissu urbain d'Épinac dans une logique duale. Cependant, cette dualité diffère de celle pouvant être observée dans d'autres villes-usine, voire certaines villes industrielles. 
27 En effet, si certaines villes-usines s'inscrivent dans une configuration urbaine marquée par une dualité prononcée, celle-ci est bien souvent matérialisée par un tissu urbain relativement dense et continu, imbriqué sans rupture sensible entre les différents quartiers concernés. Leur dualité s'opère ainsi en interaction directe, parfois même simplement délimité par un linéaire industriel (voie ferroviaire et gare de triage, canal et bassins, etc.).

Épinac possède en cela la singularité d'une dualité davantage cloisonnée, hermétique. Car si le bourg ancien et la cité minière ont fini par constituer des pôles urbains de poids comparables à la charnière des $\mathrm{XX}^{\mathrm{e}}$ et $\mathrm{XX}^{\mathrm{e}}$ siècles $(1670$ habitants recensés dans la cité de la Garenne avant 1914, pour une population communale de près de 4600 habitants), les deux tissus sont clairement distincts, séparés de près de deux kilomètres l'un de l'autre. Une dualité justifiée par la concentration des fonçages et puits de mines les plus importants en partie Est du ban.

Cette distance a ainsi constitué une coupure nette dans le paysage, encore perceptible dans les derniers temps de l'activité minière au milieu du XX $X^{\mathrm{e}}$ siècle (Figures $4 \mathrm{a}$ et $4 \mathrm{~b}$ ), confondant ainsi de manière particulièrement imbriquée les faciès urbain (village, cité, complexe minier, usines, etc) et agraire (champs, haies et pâtures).

Si Épinac a progressivement émergé comme un pôle urbain plus affirmé en accompagnement du développement de la mine (près de 5000 habitants à la veille de la Première guerre mondiale), avec notamment sa reconnaissance comme chef-lieu de canton en 1905, le bourg n'a pour autant jamais réellement rempli l'ensemble des critères d'une véritable ville, chef-lieu et complexe minier demeurant morphologiquement dos-à-dos. Une telle fragmentation dans le paysage et la morphologie urbaine de la commune a in fine dans le temps long freiné son influence et limité son poids dans la hiérarchie urbaine régionale. Se révélant en cela constitutif de sa dimension propre de petite ville-mine. Et ce malgré les velléités clairement formulées par les actionnaires des houillères par la voix de leur directeur des mines Zulma Blanchet, qui écrivit en 1876 « [qu'] il était permis de croire que lorsque le chemin de fer de Santenay à Etang-sur-Arroux sera raccordé avec la ligne d'Epinac à Velars, la houillère ne tardera pas d'être fondue avec le chef-lieu d'Epinac, du centre duquel elle est séparée par une distance de deux kilomètres. La gare de la ligne de Santenay (gare d'Epinac aujourd'hui désaffectée, sic), placée entre la Mine et Epinac, formera le trait d'union des deux localités ».

31 Cette perspective de développement, inaboutie et encore perceptible dans les paysages et la morphologie urbaine contemporaine d'Epinac (Figure 6), permet de souligner la carence évidente dans la réalisation complète du fait urbain et ainsi le manquement d'une jointure entre les deux entités d'égal poids démographique au début du XX siècle.

Épinac, même au plus fort de son développement industriel et urbain, est ainsi bien loin de former un centre urbain équivalent - aussi bien démographiquement qu'en termes de cohérence urbaine - aux autres exemples de développement industriels et miniers en Saône-et-Loire, tels que Gueugnon, Le Creusot ou encore Montceau-les-Mines. Ces derniers étant parvenu à réunir en un même lieu à la fois la capacité extractive nécessaire en termes de matière première (gisements plus conséquents que la poche géologique d'Epinac) et les sites industriels de production (verrerie et usines sidérurgiques Schneider au Creusot, forges à Gueugnon, etc). 
33 Au-delà de la genèse et de la limite spatialement constatée du développement de ce géosystème industrialo-minier, il s'agit désormais de déterminer les différentes raisons ou logiques, endogènes comme exogènes, ayant enrayé la dynamique d'émergence, pourtant souhaitée par les décideurs des houillères (Blanchet, 1876), d'une ville-mine à part entière.

Figure 4a: Croquis d'interprétation aérienne d'Épinac au milieu du XXe siècle

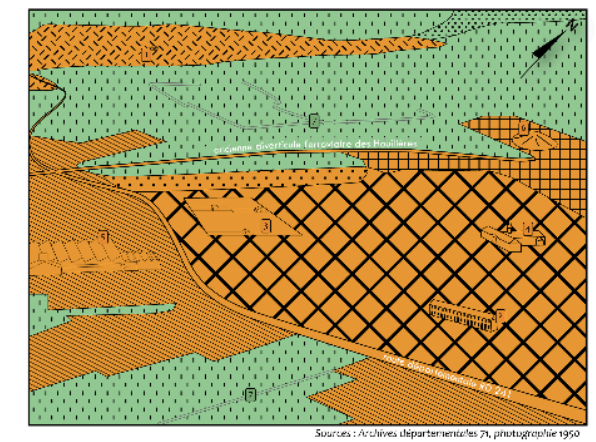

Faciès urbain.

W.:- Vieux noyaux villageois au tissu urbain semi.cisperse typique des villages du bocage bourgui-

L

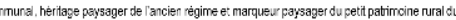
Quartier de la Cité de Garenne de la seconde moitié du XIXe siécle, aux fomes urbaines recti-

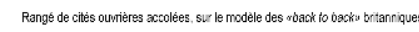

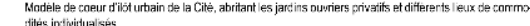

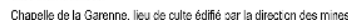

Cites des ingenieurs, placée a l'eccart de la cite ouvriere, aux habitations individuelles et davan-

tage espacées.

Quartier mitoyen à la Cité ouvr
sition urbaine plus héteroclite.

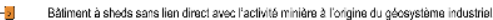

III Carreau de mine du Puits de la Garenne et ses differents bătiments de transformation et

$L_{0}$

$\square$ Faciès agrairo-rural.

$[\because \cdots$ Vastes terres agro-pastorales, dominées par l'álevage extensif.

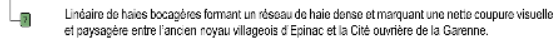

7. Massif boisé de hêtraie-chênaie, en pourtour du ban communal d'Epinac. 
Figure 4b : Cliché d'origine, pris en direction nord/nord-ouest (source : Archives départementales 71)

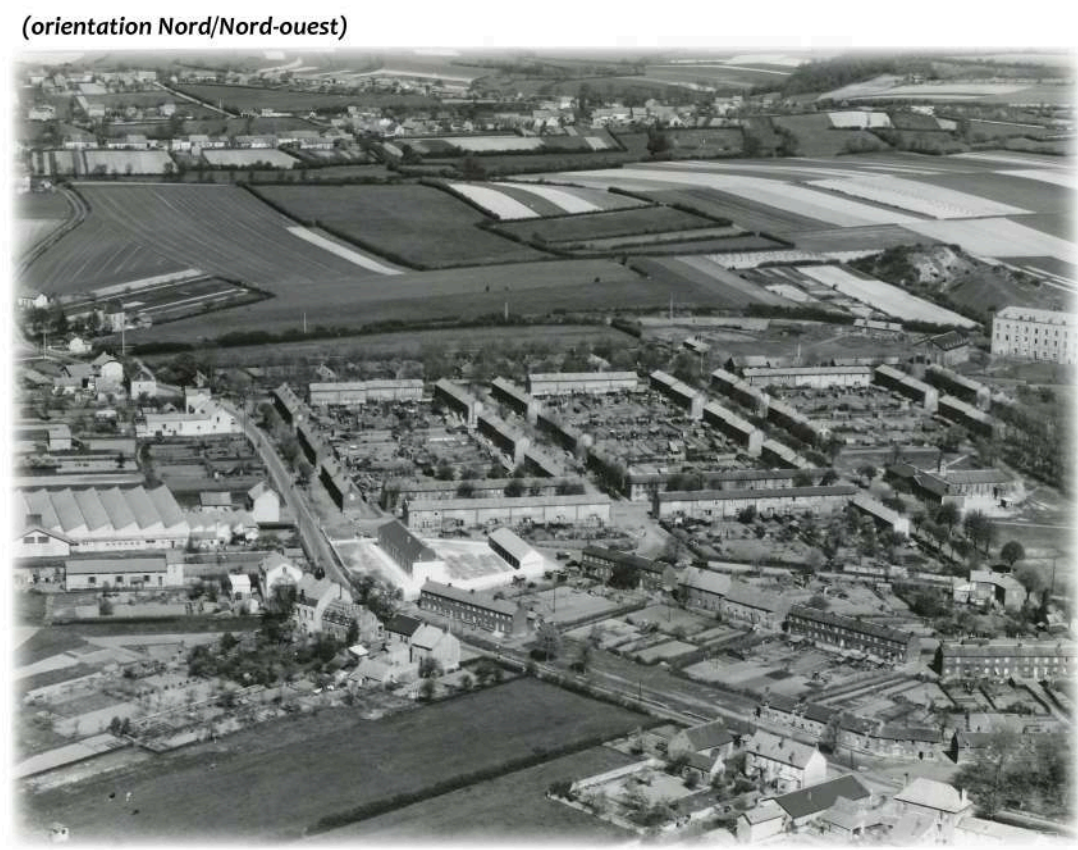

\section{Affirmation et déclin de la petite ville-mine d'Epinac : typicité ou originalité ?}

Au-delà du développement intrinsèque des houillères et leurs activités industrielles annexes liées à l'exploitation, l'insertion du système productif épinacois dans son environnement économique régional constitue, en termes de débouchés, une approche complémentaire indispensable pour comprendre l'essor (A), les limites et, in fine, le déclin connu par l'exploitation houillère à Épinac dès le début du $\mathrm{XX}^{\mathrm{e}}$ siècle (B).

À cette approche viendra s'adjoindre une mise en perspective de la dynamique de développement puis de déclin de l'activité minière sur la commune, dans une approche succincte de comparaison à d'autres communes minières. Le tout dans un contexte assimilable aux dynamiques de "ville rétrécissante ", également connues sous le terme anglais de shrinking cities (C).

\section{A. Entre complémentarité et concurrence au sein du système productif régional : les multiples composantes du développement industrialo-minier d'Epinac}

$1^{\circ}$ Expansion industrielle et débouchés économiques : aires de chalandises, principe concurrentiel et limites productives.

Du fait de son positionnement géographique, au sein de la Bourgogne et du nord-est encore peu pourvu de grands bassins miniers (l'essentiel des gisements alsaciens et mosellans passant sous nationalité allemande suite au conflit de 1870), les débouchés de production des houillères d'Épinac furent assurés, durant l'essentiel du XIX ${ }^{\mathrm{e}}$ siècle, par 
l'approvisionnement des petites unités industrielles sidérurgiques de Bourgogne (Côte d'Or avec la région du Châtillonnais et Montbard notamment) et de Franche-Comté.

Cependant, si ces débouchés ont permis aux houillères d'Épinac de garantir l'écoulement de leur production, l'absence de synergie entretenue entre les différentes exploitations et concessions minières en Saône-et-Loire (et notamment avec les puissants industriels Schneider, possédants mines et usines sidérurgiques du Creusot et s'inscrivant donc dans une logique frontalement concurrentielle) a durablement contraint les perspectives de développement (Passaqui, 2006). Cette contrainte de concurrence vis-à-vis de l'empire Schneider et de ses mines du Creusot a notamment abouti à faire avorter le projet de desserte ferroviaire depuis Epinac vers le sud et le centre du département, en direction des centres de Montceau et du Creusot, les frères Schneider s'opposant à cette réalisation qui aurait ainsi facilité l'acheminement et l'exportation du charbon d'Epinac.

Mais, au-delà même des différents impacts exogènes, Épinac a avant tout pâti, pour un développement pérenne et important de son tissu industrialo-urbain et pour le renforcement de son caractère urbain, du manque de structuration concentrée de son appareil productif ou, plus exactement, de son inachèvement.

Car si la concentration opérée au milieu du XIX ${ }^{e}$ siècle par les houillères, devenues les Houillères et Chemin de fer d'Épinac à partir de 1829 (HCE), a assuré une assise solide à l'extraction des matières premières, leur usage (verrerie un temps détenue par les possédants des houillères), leur acheminement et leur écoulement par les chemins de fer, elle n'a jamais été parachevée par l'essor d'un site sidérurgique in situ.

Hormis la verrerie (qui ferme ses portes dès 1934) de taille modeste, seule une précaire et provisoire centrale électrique, installée en lieu et place du plus imposant des puits forcés sur le ban communal, donne un temps l'illusion d'une activité amont-aval plus étoffée et à même de garantir à la ville-mine son développement urbain et industriel.

41 Cette intégration locale heurtée, les manques d'un appareil productif et de transformation qui ne soit pas davantage développé in situ, (à l'image des grands centres industrialo-urbains emblématiques du département de Saône-et-Loire, organisant une concentration des activités amont-aval) ainsi qu'un gisement houiller in fine moins prolifique que ne l'avaient estimé durant tout le XIX ${ }^{\mathrm{e}}$ siècle les différents ingénieurs des mines, ont eu pour conséquence de précipiter la fermeture des puits et de l'exploitation houillère à Épinac (dont le dernier puits est définitivement abandonné en 1966). Et ce malgré des attentes prometteuses et des investissements importants dans les moyens et techniques d'exploitations mis en œuvre.

Une autre conséquence suggérée par ces manques et insuffisances réside dans la fragilisation du tissu urbain hérité de cette époque industrielle, celui-ci se reposant quasi exclusivement sur l'activité extractive. Aucune industrie ou unité de production, aval ou satellite, n'étant en mesure de se subsister au moins partiellement à l'activité économique perdue à la fermeture du site d'exploitation.

\section{$2^{\circ}$ Déclin de l'exploitation et crise de reconversions.}

Si les années 1915 à 1919 du premier conflit mondial constituent les meilleures années d'exploitation de la concession (dépassant les 250000 tonnes de charbon extrait), la fin du conflit représente également un tournant géopolitique majeur. En effet, le retour des territoires miniers de l'Alsace-Moselle (perdus en 1870) cumulée à la fin des besoins 
de guerre rendant caduc le rythme exponentiel de croissance connu à la charnière des $\mathrm{XIX}^{\mathrm{e}}$ et XX $\mathrm{XX}^{\mathrm{e}}$ siècles marque l'apogée de l'activité extractive dans les houillères du Centre et du Midi, et particulièrement sur le gisement épinacois.

D'un point de vue morphologique, à la fermeture des derniers puits d'extraction houillère, dans l'immédiat après Seconde Guerre mondiale, la commune d'Épinac demeure fortement imprégnée par la dualité géographique de son tissu urbain. La compagnie minière n'étant pas parvenue à opérer la «couture » urbaine escomptée avec le centre-bourg d'Ancien Régime (Tillequin, 2013).

Confronté à la déliquescence continue et complète ${ }^{4}$ de l'appareil industriel et minier, la réaction de la population et des pouvoirs publics locaux s'inscrit assez fidèlement dans le schéma identifié par les sociologues dans le processus de reconversion industrielle. Ce schéma distingue trois phases d'appréhension de la disparition de l'activité (Figure 5).

La première phase - temps de l'incrédulité - se traduit territorialement par une inaction face au déclin de l'activité. Elle correspond à une certaine incompréhension devant le délitement de l'activité, partie intégrante du tissu économique, social, urbain et paysager local. Incompréhension généralement liée à l'espoir que l'activité ne traverse qu'un nouveau soubresaut économique, comme cela a déjà pu concerner de nombreuses firmes industrielles depuis la première révolution industrielle.

Figure 5 : Tableau des grandes phases de la reconversion (Edelblutte, 2014)

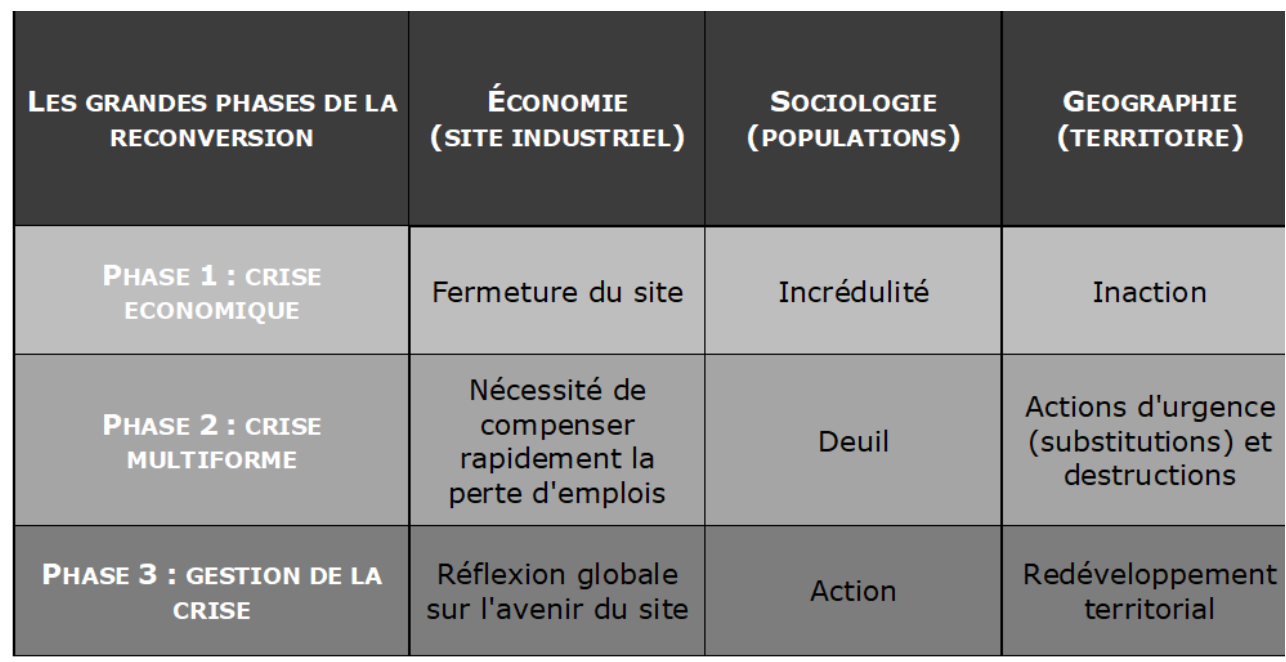

S. Edelblutte et MC Doceul

Cette première phase, quoique relativement courte dans l'histoire de l'activité industrielle et minière d'Épinac, correspond malgré tout à un temps long au regard de l'échelonnement des fermetures, échelonnées sur plus de trois décennies. Paradoxalement, la brutalité de la cessation de l'activité ne réside donc pas dans sa relative fulgurance, mais bien dans les impacts et les mutations qu'elle implique, pour un territoire façonné depuis près de 200 ans par et pour l'activité verrière et minière. 
48 La deuxième phase déterminée par les sociologues - le temps du deuil - se traduit spatialement par la mise en place de premières actions en réaction au déclin de l'activité ancienne. Ces actions sont principalement de deux natures :

- Le remplacement de la perte d'activité par l'implantation de nouvelles activités, le plus souvent sans réflexion d'ensemble ou de long terme;

- La destruction des marqueurs paysagers et symboles rappelant l'activité historique déchue.

49 Ce temps, par la progressivité du déclin de l'activité ne concerne cependant pas l'ensemble des sites et lieux de la production de manière homogène. Ainsi, la reconversion du site du puits Hottinguer, fleuron architectural et technologique des houillères et marqueur paysager parmi les plus imposants de l'activité minière du bassin d'Autun, est engagée dès 1947. La friche du puits de mine, et notamment sa Tour Malakoff, est reprise et reconverties par l'entreprise Bitulac ${ }^{5}$.

Celle-ci produit des peintures, vernis, enduits spéciaux et matériaux isolants. L'entreprise, prospère, emploie rapidement plus d'une centaine de salariés, offrant ainsi une solution d'emploi in situ pour une partie des anciens ouvriers et mineurs restés sur place.

51 Cette stratégie de reconversion articulée autour d'une nouvelle entreprise prenant le relai de l'ancienne activité se retrouve dans le cas d'autres petites villes-mine. C'est notamment le cas de Saint-Eloy-les-Mines, dont la reconversion post-minière s'est elle aussi concentrée autour d'une seule usine, avec l'entreprise Rockwool. Cette reconversion vient ainsi prolonger les risques liés à la mono-activité, avec un manque de résilience ayant déjà pesé sur ces territoires durant la phase d'exploitation minière, activité omniprésence.

52 L'ensemble du patrimoine bâti local lié à la mine est également rapidement délaissé et détruit (chevalements, fours à coke, laminoirs, triages...). C'est ainsi le cas de l'essentiel des puits et de leur chevalement, dont les bâtiments et installations, disséminés sur le ban communal, sont d'autant plus aisés à effacer du paysage que ceux-ci sont diffus.

53 L'habitat ouvrier fait également l'objet de destructions précoces. Dès les années 1950, la vacance importante résultante du déclin de l'activité et du départ de familles de mineurs pour d'autres sites industrialo-miniers en Bourgogne à Autun, Montceau, ou en Lorraine à Carling, Farébersviller (Darroux et Guillaumeau, 2013), une partie de la Cité de la Garenne (l'extrémité sud-est) est détruite. Elle est remplacée par plusieurs barres de logements collectifs typiques des années 1950-60, censés répondre aux nouvelles exigences en matière de confort.

54 Dans la même approche des politiques d'habitat et d'urbanisme, les années 1970 et 1980 voient émerger la tentative d'une jointure urbaine entre le bourg médiéval et la Cité minière, par la création d'un vaste lotissement pavillonnaire, auquel s'agrège quelques collectifs et différents équipements et services (caserne de pompiers, équipements sportifs, supermarché...). Le tout formant un patchwork urbain peu lisible et ne parvenant pas réellement à créer un trait d'union cohérent entre les deux tissus préexistants (Figure 2).

55 Ces différentes actions, hâtives et sans réflexion d'ensemble sur la reconversion du tissu économique et social local ne répondent ainsi que de façon palliative à la crise, et n'enrayent en rien le déclin démographique et de rayonnement de la petite ville-mine sur son environnement local. 


\section{B. Une démographie de « ville-rétrécissante » ?}

56 A l'instar de villes et métropoles largement connues et étudiées (Détroit, Liverpool, ...) les communes de taille démographique plus modeste comme Épinac recouvrent les mêmes tendances de rétrécissement.

Conceptualisé autour du terme de "shrinking cities" ou villes rétrécissantes, ce phénomène, observable sur un certain nombre de villes proches du bassin d'Epinac (les villes d'Autun, du Creusot et de Montceau-les-Mines par exemple) peut se définir, d'après le site Géoconfluences ${ }^{6}$ comme " un phénomène de rétrécissement urbain qui touche les villes sur trois plans : démographique, par la perte de population ; économique, par la perte d'activités, de fonctions, de revenus et d'emplois ; et social, par le développement de la pauvreté urbaine, du chômage et de l'insécurité ».

58 A ces trois premiers plans d'analyse peut en être adjoint un quatrième: le plan géographique, l'impact du phénomène décrit engendrant de multiples mutations du tissu urbain, tant dans son enveloppe urbaine (friches urbaines, logements vacants, ruines ou "dents creuses », ...) que dans sa dynamique de développement (étalement urbain, nouvelles centralités, déficit d'image, ...). "Les shrinking cities sont [ainsi] associées au "déclin urbain" ou encore à la "décroissance urbaine" des villes industrielles » (Fol et Cunningham-Sabot, 2010 ; Wolff, Fol, Roth et Cunningham-Sabot, 2013).

Or, si ce phénomène s'applique en premier lieu aux villes industrielles, il n'en demeure pas moins reconnaissable dans les caractéristiques démographiques, économiques et sociales du déclin de l'activité industrielle et de son impact sur le tissu urbain des petites villes-usines et petites villes-mines issues de géosystèmes industriels et miniers planifiés (chalard, 2007).

60 Il en va ainsi des nombreuses petites villes-mines issues de l'exploitation minière $d u$ Massif central et du Midi, ces centres urbains ayant connu un essor démographique comparable, tant par leurs bornes temporelles que par leur ampleur. Ainsi est-il utile, afin d'identifier les dynamiques caractéristiques et de les distinguer des phénomènes plus particuliers, d'établir une brève étude comparative des résultantes démographiques de l'essor, du déclin puis de l'arrêt de l'activité minière à l'échelle de plusieurs petites villes-mines françaises.

61 Pour étayer cette analyse, les principales communes des cinq bassins miniers du Massif central et ses bordures ont été retenues, étant inscrites dans des bassins d'exploitation sensiblement comparables dans leur dimension et leur importance d'extraction (Figure 6) : La Machine, Blanzy, Montceau-les-Mines, Epinac (Houillères de Blanzy), Buxièresles-Mines, Noyant d'Allier, Saint-Eloy-les-Mines, Messeix, Brassac-les-Mines (Houillères d'Auvergne), Saint-Martin-de-Valgalgues, La Grande Combe (Houillères des Cévennes), Decazeville, Carmaux (Houillères du Midi) Rive de Gier et Firminy (Houillères de la Loire). 
Figure 6 : Evolution démographique comparée d'Epinac avec les principales communes minières du Massif central et ses bordures (en base 100 pour 1793, ou l'année de création de la commune pour les communes nouvelles)

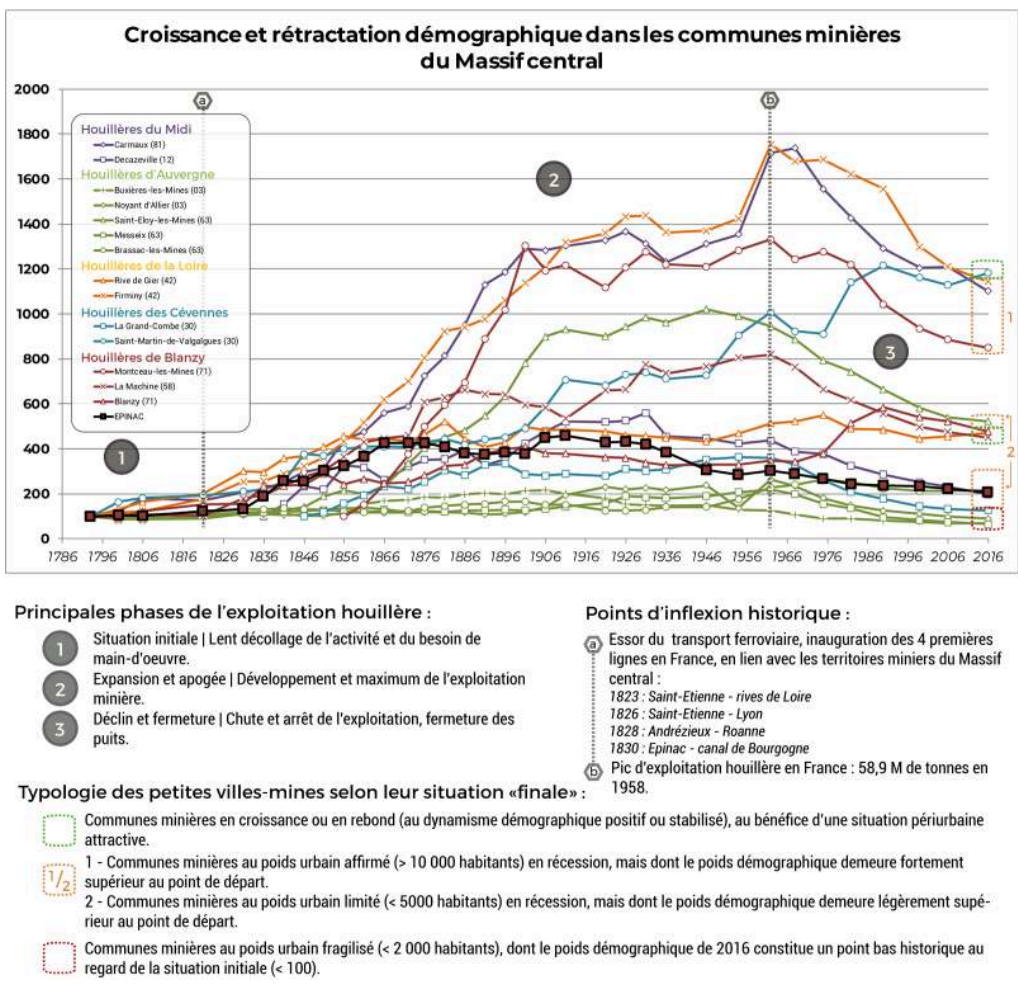

Le graphique en Figure 6 représente la dynamique démographique de chacune de ces communes depuis la fin du XVIII ${ }^{e}$ siècle jusqu'au début du XXI ${ }^{e}$ siècle, en base 100. Cette analyse, concentrée sur la seule échelle communale, permet néanmoins de dégager plusieurs tendances pour ces différentes villes-mines du Massif central :

- Une situation initiale (fin XVIII / début XIX ${ }^{e}$ siècle) sensiblement comparable entre ces différentes communes. Toutes nées ex-nihilo ou d'un tissu urbain préexistant inférieur à 2000 habitants, cette phase se matérialise autour d'un lent décollage de l'activité extractive.

- Une deuxième phase d'expansion et d'apogée, caractérisée par la morphologie de ces courbes démographiques, similaire pour la majorité de ces communes, suivant une trajectoire ascendante. Des disparités s'observent néanmoins dans la plage temporelle comme dans l'intensité de l'expansion démographique, ainsi que, par voie de conséquence, dans la date d'atteinte de l'apogée de chaque commune (1911 pour Epinac, 1931 pour Decazeville, 1962 pour Montceau-les-Mines).

- Une dernière phase ou situation finale elle aussi grandement comparable entre ces territoires, les communes enregistrant sur les dernières décennies une importante décroissance de leur population. Malgré cela, elles se maintiennent dans la majorité des situations à un poids démographique sensiblement supérieur à leur situation initiale. Quatre communes se distinguent néanmoins, par deux formes distinctes :

- Les communes de Messeix, Noyant d'Allier et Buxières-les-Mines, dont la situation finale s'inscrit bien en deçà de leur point de départ de 1793, avec un poids démographique de seulement d'à peine 1000 habitants ;

- La commune de Saint-Martin-de-Valgalgues qui connait, malgré l'arrêt de l'activité minière, un nouveau dynamisme démographique en grande partie dû à sa situation géographique (développement périurbain d'Alès). 

fonction de l'intensité de leur courbe parabolique ainsi que du niveau démographique au point d'arrivée :

- les communes dont la démographie enregistre une croissance ou un rebond depuis la seconde moitié du XXe siècle (en vert sur la situation finale de la Figure 6), bénéficiant d'une situation territoriale attractive en périphérie d'agglomération, par un phénomène de périurbanisation compensant les pertes initiales d'actifs de la mine.

- les communes dont le poids démographique est supérieur à 10000 habitants (en orange n ${ }^{\circ} 1$ sur la situation finale de la Figure 6); celles-ci sont toutes dans une dynamique récessive, mais conserve malgré tout un poids territorial, administratif et économique certain dans leur environnement local voire régional.

- les communes dont le poids démographique est limité ( $<5000$ habitants) et dont la dynamique est structurellement récessive, mais conservant un poids démographique légèrement supérieur à leur point de référence initial (en orange $\mathrm{n}^{\circ} 2$ sur la situation finale de la Figure 6)

- les communes au poids démographique fragilisé (en rouge sur la situation finale de la Figure 6), inférieur à 2000 habitants, et dont la démographie, structurellement récessive depuis la fin du XXe siècle, aboutie à une situation finale inférieure au point de référence initial.

Au regard de ces distinctions typologiques, Epinac constitue un modèle de commune minière de catégorie intermédiaire, au poids démographique limité (inférieur à 5000 habitants) et fleurtant même avec le seuil aussi symbolique que fatidique des 2000 habitants nécessaires pour être considérée comme commune urbaine au sens de 
l'INSSE. Sa population ayant continuellement diminuée depuis les années 1950, Epinac ne compte plus que 227 habitants pour l'année 2016 (contre 3120 au recensement de 1968).

\section{Conclusion}

Fruit de l'émergence des progrès et des développements techniques depuis le milieu du XVIII $^{\mathrm{e}}$ siècle en France et la première révolution industrielle, dont le modèle économique s'est progressivement articulé autour du charbon, Épinac a connu un essor industriel certain. Essor conjuguant l'installation de branches industrielles liées entre elles : verrerie, houillères et chemins de fer.

Le développement concomitant de ces différents domaines nécessaires à la production a engendré de nombreux bouleversements tout au long du XIX ${ }^{\mathrm{e}}$ siècle, le tissu urbain et l'ensemble du ban communal d'Épinac connaissant de profondes mutations de l'occupation des sols et de ses morphologies paysagère et urbaine. Ce développement industrialo-minier a ainsi transformé, par la forte croissance démographique en résultant (solde migratoire), l'ancien petit bourg médiéval en une véritable petite villemine, lui conférant même jusqu'au statut administratif de chef-lieu de canton au début $\mathrm{du} \mathrm{XX}^{\mathrm{e}}$ siècle.

71 Suite aux grandes crises géopolitiques et industrielles ayant émaillées le $\mathrm{XX}^{\mathrm{e}}$ siècle, l'ensemble du tissu industrialo-minier présent sur le ban d'Épinac, à l'instar des grands bastions miniers et sidérurgiques du département, cesse toute activité en l'espace de quelques décennies. Le choc économique et social, difficilement appréhendé par les pouvoirs publics, aboutit à des initiatives de reconversions privées au coup-par-coup, connaissant des fortunes diverses.

Cependant, ni le travail de reconversion du tissu économique local, ni l'aménagement urbain d'un nouveau quartier résidentiel voué à réaliser la jonction entre le vieux village et la cité minière - celle-la-même que les dirigeants des houillères du XIX ${ }^{\mathrm{e}}$ siècle entendaient opérer - n'ont permis d'enrayer la chute démographique continue engendrée par la fermeture des activités industrielles et minières (Figure 6).

73 Par l'importance de son essor comme par les multiples facettes de son déclin et ses reconversions, le développement de l'activité industrialo-minière d'Épinac constituent ainsi un objet géographique révélateur des tendances et dynamiques territoriales propres aux géosystèmes industriels planifiés, au développement demeuré incomplet ou de rayonnement urbain limité. Le cas d'Epinac illustre bien, au même titre que d'autres petites villes-mines évoquées précédemment (Saint-Eloy-les-Mines, La Machine...), que la planification urbaine et paternaliste des industriels du XIX ${ }^{\mathrm{e}}$ siècle n'a pas systématiquement abouti à l'émergence d'une ville-mine capable de muter, une fois l'activité disparue, en une ville à part entière, résiliente et à même de développer et d'assurer de nouvelles fonctions urbaines. 


\section{BIBLIOGRAPHIE}

Benoît R., 2013, « Le P.L.U et le patrimoine industriel de la ville d'Épinac », Actes du Colloque Patrimoine industriel des petites villes, Guérigny, éd. Centre d'Etudes de la métallurgie nivernaise, $p$. 213-218.

Bergeron L. et Dorel-Ferré G., 1996, Le patrimoine industriel : un nouveau territoire, Paris, éd. Liris, $127 \mathrm{p}$.

Blanchet Z., 1867, L'exploitation de la Houille à Épinac, imprimerie M.Dejussieu, Autun, 269p.

Blanchet Z., 1872, L'exploitation de la Houille aux grandes profondeurs : Puits Hottinguer de la S.A. des Houillères et Chemin de fer d'Épinac, imprimerie M.Dejussieu, Autun, 21p.

Chalard L., 2007, « Des villes françaises en net déclin démographique. Le cas de communes anciennement industrielles ", Population et Avenir, $n^{\circ}$ 683, p.17-19.

Darroux C. et Guillaumeau R., 2013, L'Or Noir, la vie au temps de la mine dans la vallée de la Drée, édité par la Communauté de Communes de la Vallée de la Drée et la Maison du patrimoine oral de Bourgogne, 99p.

Deshaies M., 2007, Les territoires miniers, exploitation et reconquête, Paris, Ellipses, 224p.

Del Biondo L., Edelblutte S., 2016, Le paysage des anciennes villes-usines européennes : un nouveau patrimoine entre négation, alibi, reconnaissance, et complexité des jeux d'acteurs, Annales de Géographie, $n^{\circ} 711$, p.466-489.

Dorel-Ferré G. et als., 2003, La cité-jardin : une histoire ancienne, une idée d'avenir, éd. Canopé, CRDP de Reims, Coll. Patrimoine ressources, 156 p.

Dorel-Ferré G., Varashin D. et als., 2016, Villages ouvriers et villes-usines à travers le monde, Université de Savoie, 288p.

Doumergue Y., 1964, Un bel exemple d'implantation industrielle en milieu rural : l'usine Bata de MousseyBataville (Moselle), L'information géographique, volume 28, n³, 1964. pp. 125-130.

Edelblutte S., 1998, «Genèse et mutation d'un géosystème industriel, l'exemple de NeuvesMaisons, Côte de Moselle », Revue Géographique de l'Est, tome 38, n¹-2, 1998. Géohistoire des paysages des côtes de Lorraine et du Luxembourg, p. 31-49.

Edelblutte S., 2009, Paysages et territoires de l'industrie en Europe : héritages et renouveaux, Paris, Ellipses, $272 \mathrm{p}$.

Edelblutte S., 2010, La reconversion des anciennes villes-usines européennes ou la question de la survie urbaine, Géographies, Bulletin de l'Association de Géographes Français, n³, p. 353-367.

Edelblutte S. et Legrand J., 2012, « Patrimoine et culture industriels en milieu rural : quelles spécificités? », Revue Géographique de l'Est, vol. 52 / 3-4 | 2012.

Edelblutte S., 2014, « Reconversion industrielle ou redéveloppement territorial ? L'exemple de Thaon-les-Vosges, ancienne ville-usine textile lorraine », Géoconfluences, 2014.

Fol, Sylvie, et Emmanuèle C. Cunningham-Sabot. « « Déclin urbain » et Shrinking Cities : une évaluation critique des approches de la décroissance urbaine ", Annales de géographie, vol. 674, no. 4, 2010, p. 359-383. 
Letrange Y., 2013, L'or Noir et la mémoire minière : mise en valeur par la vallée de la Drée et la Maison du Patrimoine Oral, Revue Vents du Morvan, n49, p.46-53.

Passaqui J-Ph., Chabard D., 2007, Les routes de l'énergie : Épinac-Autun-Morvan, patrimoine industriel, scientifique et technique, des ressources minérales aux richesses patrimoniales, Autun, Muséum d'Histoire Naturelle, 2007, 152p.

Passaqui, J-Ph., (2006), La stratégie des Schneider : Du marché à la firme intégrée (1836-1914), Rennes, Presses universitaires de Rennes, 2006, 410p.

Simonin L., (1867), La vie souterraine, ou Les Mines et les mineurs, L. Hachette et Cie, Paris, $2^{\mathrm{e}}$ édition, 1 vol. III. $607 \mathrm{p}$.

Tillequin S., 2013, « Le chemin de fer d'Épinac et le quartier de la Gare », Actes du Colloque Patrimoine industriel des petites villes, Les Amis du Vieux Guérigny, Guérigny, éd. Centre d'Etudes de la métallurgie nivernaise, p.203-212.

Varine (de) H., 2013, «Quels sont les choix, qui les fait et pourquoi : destruction, conservation, transformation, réutilisation?", Actes du Colloque Patrimoine industriel des petites villes, Les Amis du Vieux Guérigny, Guérigny, éd. Centre d'Etudes de la métallurgie nivernaise, p.63-74.

Wolff M., Fol S., Roth H. et Cunningham-Sabot E., 2013, « Shrinking Cities, villes en décroissance : une mesure du phénomène en France ", Cybergeo : European Journal of Geography [En ligne], Aménagement, Urbanisme, document 661, mis en ligne le 08 décembre 2013, consulté le 14 avril 2018, URL : http://journals.openedition.org/cybergeo/26136

Woronoff D., 1994, Histoire de l'industrie en France, du XVI siècle à nos jours, Paris, Le Seuil, 674 p.

\section{NOTES}

1. «Un géosystème est un territoire auquel on peut fixer une limite et qui s'organise fonctionnellement autour d'un centre " (A.Humbert, 1994). Le terme de géosystème est ici employé dans une acception restreinte où l'ensemble des marqueurs territoriaux et paysagers induits, de manière directe (chevalements, lavoirs, usines, chemins de fer, ...) comme indirecte (cités, coopératives, écoles, infirmerie, cantine, bibliothèque, ...), construit un système spatial original et cohérent, centré sur l'activité industrielle ou minière in situ.

2. http://geoconfluences.ens-lyon.fr/actualites/veille/villes-retrecissantes-renouveau

3. Ces termes sont préférés à ceux de ville industrielle ou ville minière, qui impliquent une existence préalable de la ville, du fait urbain, aux activités industrielles et minières.

4. Disparition totale de l'ensemble des emplois de la verrerie, dont l'activité multiséculaire remontait au milieu du XVIII ${ }^{e}$ siècle, des houillères et du chemin de fer.

5. Les produits de la société Bitulac sont notamment utilisés dans la conception du navire Charles de Gaulle, du pont de Tancarville, de rames TGV, du tunnel sous la Manche ou encore du Stade de France.

6. http://geoconfluences.ens-lyon.fr/glossaire/shrinking-city

7. D'après les dernières estimations de l'INSEE, Epinac comptait pour l'année 20162227 habitants. 


\section{RÉSUMÉS}

Située sur la bordure septentrionale du Massif central, Epinac est une commune bourguignonne dont la morphologie urbaine et les paysages hérités représentent un cas particulièrement intéressant de développement industriel et minier en milieu rural.

La concession minière développée à Épinac s'inscrit dans le cadre géographique plus large des houillères du bassin de Blanzy, bassin dont les grands centres industrialo-urbains du Creusot et de Montceau-les-Mines voisins constituent encore aujourd'hui les principales agglomérations industrielles de la nouvelle région Bourgogne-Franche-Comté.

Les impacts et les mutations impliqués par ce développement industrialo-minier à l'échelle de la commune, à la fois socio-économiques et paysagers, ont été conjointement et progressivement établis par l'essor conjugué des activités du verre, de la houille et du chemin de fer.

Ainsi durant plus de deux siècles (de la mi-XVIII ${ }^{\text {ème }}$ à la mi-XX ${ }^{\text {ème }}$ siècle), l'essor de ces différentes activités a été à l'origine d'un paysage et d'une structuration territoriale assimilables par bien des égards aux tissus industrialo-urbains, quoique les manques et carences pour parfaire le développement urbain et démographique d'Epinac constituent des révélateurs essentiels des limites d'un développement industrialo-urbain planifié.

L'arrêt et la fermeture, au mitan du XX ${ }^{\text {ème }}$ siècle, de l'ensemble des sphères productives "historiques" du développement territorial d'Epinac ont faitémerger de nouveaux enjeux territoriaux, parmi lesquels la gestion de la crise puis les reconversions et redéploiement du tissu industriel, forment les premières étapes de l'après-mine et de la recherche d'une nouvelle identité et d'un nouveau positionnement au sein du réseau urbain régional.

Located at the northern border of the Massif central, Épinac is a burgundian township whose urban morphology and inherited landscape constitute a particularly interesting case of industrial and mining development in rural areas.

The mining site of Epinac it is located in the geographical environment to the basin of Blanzy, whose Épinac mining concession is include. In this context, the most important industrial cities in this area are Creusot and Monceau-les-Mines and they remains at this time one of the main industrial places of the Bourgogne-Franche-Comté new region.

Impacts and mutations involved by this industrial and mining development at the scale of the town, as much socio-economical than on the landcape, have been jointly and progressively established during the mutual peak in the glass, coal and railway's activities.

Therefore, during more than 2 centuries (from mid-1700's to mid-1900's), the apparition of these different activities has been at the origin of a landscape and a territorial structuration assimilable by many means at industrial and urban fabrics, even if the carences and shortages to perfectionnate the Epinac's urban and demographic development represent essential indicators of planned industrial and mining development's limits.

The global end and closing, in the middle 20th century, of historical production's sphere's of Epinac's territorial development have made appeared new territorial aims, such as crisis' gestion or industrial fabrics's reconversion and repositioning, which form first steps of the post-mining era and of the research toward a new identity and a new positionment inside the regional urban's network.

Epinac, die am nördlichen Rand des Massif central liegt, ist eine burgundische Gemeinde, deren Stadtmorphologie und Erblandschaften ein hochinteressantes Fallbeispiel der industriellen und bergbaulichen Entwicklung in einer ländlichen Umgebung darstellen.

Die in Epinac entwickelte Bergbauberechtigung lässt sich im breiteren geographischen Rahmen der Kohlengruben des Bergbaureviers von Blanzy eingliedern, in welchem die angrenzenden 
großen industriellen und städtischen Zentren von dem Creusot und Monceau-les-Mines heute noch die bedeutendsten industriellen Großräume der neuen Region Bourgogne-Franche-Comté bilden.

Die sozioökonomischen sowie landschaftlichen Wirkungen und Umbrüche, die von dieser industriellen und bergbaulichen Entwicklung verursacht worden sind, wurden gemeinsam und schrittweise vom vereinten Aufschwung des Glas-, Kohle- und Eisenbahnbetriebs erstellt.

Mehr als zwei Jahrhunderte lang (von der Mitte des 18. Jahrhunderts bis zur Mitte des 20. Jahrhunderts) fuhr der Aufschwung dieses vielseitigen Betriebs daher zu einer Landschaft und einem territorialen Aufbau, die in vielerlei Hinsicht mit den industriellen und städtischen Geweben gleichgestellt werden können. Immerhin stellen die Mängel und Unzulänglichkeiten hinsichtlich der Vollendung der städtischen und demographischen Entwicklung Epinacs wesentliche Indikatoren für die Grenzen einer geplanten industriellen und städtischen Entwicklung dar.

In der Mitte des 20. Jahrhunderts fuhren der Verschluss und die Schließung aller „historischen“ Produktionsbereiche der territorialen Entwicklung Epinacs zum Auftauchen neuer territorialen Herausforderungen. Unter denen bilden das Krisenmanagement und später die Umstellungen und die Entfaltung des industriellen Gewebes die ersten Stufen der Nachbergwerkszeit und der Suche nach einer neuen Identität und einer neuen Stelle innerhalb des regionalen städtischen Netzes.

INDEX

Mots-clés : industrie en milieu rural, révolutions industrielles, reconversion, mines, verrerie, chemin de fer, géosystème industriel, paternalisme, patrimoine, héritages

Keywords : industry in rural area, industrial revolutions, industrial conversion, mines, glass factorie, railway, industrial geosystem, paternalism, heritage

Schlüsselwörter : Industrie in ländlicher Umgebung, industrielle Revolution, Umstellung, Bergwerke, Glasfabrik, Eisenbahnlinie, industrielles Geosystem, Paternalismus, Vermögen, Erbe

\section{AUTEUR}

\section{LOMAN GAUDUCHON}

Enseignant en histoire-géographie - Collège Louis Armand, Golbey (Vosges) - Université de Lorraine - Laboratoire LOTERR - loman.gauduchon@ac-nancy-metz.fr 